\title{
Factores deteriorantes de la calidad de vida en insuficiencia cardiaca: revisión integrativa
}

\section{Deteriorating factors in quality of life in heart failure: integrative review}

\author{
Diana Molano-Barrera (iD) ${ }^{1}$, Renata González-Consuegra (iD) ${ }^{2}$
}

1. Universidad Nacional de Colombia. Bogotá, Colombia. Correo: dymolanob@unal.edu.co - http://orcid.org/0000-0001-7579-1159

2. Universidad Nacional de Colombia. Bogotá, Colombia. Correo: rvgonzalezc@unal.edu.co - http://orcid.org/0000-0001-9269-7789

Tipología: Artículo de revisión

Para citar este artículo: Molano-Barrera D, González-Consuegra R. Factores deteriorantes de la calidad de vida en insuficiencia cardiaca: revisión integrativa. Duazary. 2021 enero; 18(1): 86-98. Doi: https://doi.org/10.21676/2389783X.3889

Recibido en julio 21 de 2019

Aceptado en agosto 26 de 2020

Publicado en línea en enero 07 de 2021

Palabras

clave:

calidad de

vida;

insuficiencia

cardiaca; condiciones sociales; depresión.

\section{RESUMEN}

La insuficiencia cardiaca es una enfermedad que aumenta la morbimortalidad, deteriora la calidad de vida de la persona que la padece e impacta negativamente en el sistema de salud. El propósito de esta revisión es explorar la literatura para determinar qué condiciones biopsicosociales del individuo pueden ser factores deteriorantes de la calidad de vida relacionada con la salud en esta población. Se realizó una revisión integrativa a partir de artículos publicados entre el 2014 y el 2018, en idioma español e inglés, los cuales se obtuvieron de la revisión de las bases de datos Ovid, Scopus, Scielo, Science direct, Redalyc, Lilacs, Dialnet y Pubmed, utilizando los descriptores de consulta Mesh: quality of life and heart failure. Se incluyeron en la revisión 24 artículos, en los cuales se identificaron 24 factores deteriorantes que fueron clasificados en las dimensiones biopsicosociales del individuo. Determinar la presencia de estos factores permite al profesional de salud obtener elementos claves para el establecimiento de un plan de atención individualizado que genere un impacto positivo en la condición de salud y en la calidad de vida de la persona. Esto exige un mayor desarrollo investigativo que permita conocer este fenómeno en el contexto colombiano y latinoamericano.

\section{ABSTRACT}

Keywords: Quality of Life; Heart Failure; Social Conditions; Depression.
Heart failure is a disease that increases morbidity and mortality, deteriorates the quality of life of the people who suffer it, and negatively impacts the health system. The purpose of this review is to explore the literature to determine what biopsychosocial conditions of the individual may be deteriorating factors in health-related quality of life in this population. An integrative review was carried out based on articles published between 2014 and 2018, in Spanish and English, which were obtained from the review of Ovid, Scopus, Scielo, Science direct, Redalyc, Lilacs, Dialnet, and Pubmed databases, using the Mesh query descriptors: quality of life and heart failure. Twenty four articles were included in this review, where twenty-four factors were identified and classified in the biopsychosocial dimensions of the individual. Determining the presence of these factors allows health professionals to obtain key elements for the establishment of an individualized care plan that generates a positive impact on the person's health condition and quality of life. More research is needed to understand this phenomenon in the Colombian and Latin American context. 


\section{INTRODUCCIÓN}

La Insuficiencia Cardiaca (IC) es una alteración de la función ventricular, más la presencia de signos y síntomas asociados a la disfunción mecánica o estructural de uno o ambos ventrículos. Su etiología puede clasificarse en isquémica y no isquémica, encontrando dentro del segundo grupo las miocardiopatías familiares, las enfermedades valvulares, la hipertensión arterial, las enfermedades del pericardio, endocárdicas, congénitas, las alteraciones del ritmo cardiaco, entre otras ${ }^{1}$. Esta enfermedad tiene una alta prevalencia en la población adulta, con un importante impacto en la morbimortalidad, estimándose para Colombia en 2,3\%, lo que afecta en mayor medida a las personas con edad superior a los 70 años $^{2,3}$. Esta mayor incidencia se relaciona con el envejecimiento de la población y el incremento en los factores de riesgo cardiovascular, tales como el sedentarismo, los hábitos en el estilo de vida, la dieta y los factores culturales. Finalmente, la IC impacta al sistema de salud al generar una demanda frecuente de los servicios de atención en salud, un incremento en la tasa de hospitalizaciones $y$ el uso de tratamientos terapéuticos de alto costo que consumen los recursos del sistema ${ }^{1}$.

La persona con IC se ve enfrentada a una serie de cambios en su estado físico, emocional y sociofamiliar que dificulta la capacidad de adaptación a la nueva situación de salud y que, en consecuencia, afecta negativamente su Calidad de Vida Relacionada con la Salud (CVRS), en comparación con otras enfermedades crónicas ${ }^{4-6}$. EI deterioro de la CVRS varía de acuerdo con las características propias del individuo tales como su edad, el sexo, la presencia de fragilidad y las comorbilidades asociadas; así como el estado de la IC, la capacidad funcional, la Fracción de Eyección del Ventrículo Izquierdo (FEVI), la duración y gravedad de la enfermedad, entre otros; y condiciones psicosociales tales como las características socioeconómicas, la disponibilidad de apoyo familiar y social, y la presencia de ansiedad, depresión o deterioro cognitivo.
Al ser la calidad de vida un concepto abstracto que puede ser visto desde diferentes enfoques, es preciso concretarlo vinculándolo con la experiencia de la salud humana. La CVRS es un concepto multidimensional que incluye componentes físicos, emocionales y sociales asociados con la enfermedad". Según Urzúa ${ }^{8}$, la calidad de vida es "el nivel de bienestar derivado de la evaluación que la persona realiza de diversos dominios de su vida, considerando el impacto que en estos tiene su estado de salud". Bredow y Peterson ${ }^{9}$ la definen como un "subconjunto de calidad de vida que representa sentimientos, actitudes o la habilidad para experimentar satisfacción en el área de la vida, que ha sido interrumpido por procesos de enfermedad o déficits relacionados con la salud".

En este contexto, la determinación de la CVRS y los factores que influyen sobre esta adquieren importancia, pues contribuyen a identificar las prioridades, orientar la planificación y el desarrollo de programas preventivos, las intervenciones y la organización de servicios sociales y de salud que van más allá de la atención convencional y sirven de insumo para la formulación de objetivos, guías y políticas en salud ${ }^{7,8,10}$. Esto se verá reflejado en el logro de un tratamiento médico óptimo, mejor salud física y psicosocial del individuo, y resultados positivos para la sociedad, siendo un indicador de resultado. Es necesario identificar, en primer lugar, cuáles son los factores que influyen en su deterioro, por lo cual, fue de interés para las investigadoras determinar qué condiciones biopsicosociales del individuo pueden ser factores deteriorantes de la CVRS en pacientes con IC, siendo ese el objetivo de la presente revisión integrativa.

\section{MATERIALES Y MÉTODOS}

\section{Tipo de investigación}

Se utilizó la revisión integrativa como metodología de investigación para realizar una síntesis de los estudios publicados y establecer conclusiones generales ${ }^{11}$ respecto a los factores deteriorantes de la calidad de vida de la persona con IC. Para el desarrollo de la revisión se planteó la pregunta ¿Cuáles son las condiciones biopsicosociales de la persona con IC que son factores deteriorantes de la 
CVRS?, se realizó una delimitación de tiempo del 2014 al 2018, definiendo como palabras clave para la búsqueda "insuficiencia cardiaca" y "calidad de vida", en español e inglés, haciendo uso de los operadores booleanos para la ecuación de búsqueda [quality of life] and [heart failure]. Se utilizaron las bases de datos Ovid, Scopus, Scielo, Science Direct, Redalyc, Lilacs, Dialnet y Pubmed.

Hicieron parte de la revisión los artículos de investigación que determinaron la calidad de vida del paciente con IC y la relación con factores que ocasionaban su deterioro. Se excluyeron de la búsqueda artículos de revisión, metaanálisis, estudios de caso y estudios que incluyeran población pediátrica y pacientes en tratamiento paliativo, por requerir un abordaje multidisciplinario específico con reorientación terapéutica cuando la enfermedad entra a fase avanzada. También se excluyeron cuidadores, pues la experiencia del cuidador no se contempla dentro las condiciones biopsicosociales del individuo; los artículos que evaluaron instrumentos de medición de la CVRS y, finalmente, aquellos que evaluaron la CVRS posterior a intervenciones de tipo educativo, tratamiento farmacológico e intervenciones terapéuticas, pues su objetivo fue evaluar el impacto de la intervención y no determinar los factores que son de interés para la presente revisión. Para la lectura crítica de los artículos preseleccionados se utilizó la declaración STROBE ${ }^{12}$, teniendo en cuenta que el total de los artículos identificados eran de tipo observacional. Los hallazgos de los artículos seleccionados se sistematizaron en una base de datos, organizándolos de acuerdo con sus características, metodología, resultados y conclusiones. Los factores identificados se categorizaron en tres dimensiones, lo que facilito su análisis y la presentación de resultados y conclusiones.

\section{RESULTADOS}

En el proceso de selección se identificaron 223 artículos relevantes, de los cuales fueron rechazados 178 por cumplir con los criterios de exclusión definidos. Se preseleccionaron 45 artículos a los cuales se les realizo una lectura crítica haciendo uso de la declaración de STROBE, revisando la claridad de la información planteada en cada artículo en las secciones de título, resumen, introducción, métodos, resultados y discusión. Se seleccionaron para la revisión 24 artículos.

Los artículos seleccionados (Tabla 1) proceden de los continentes americano, europeo, asiático y australiano, representados por los países de Brasil, Estados Unidos, España, Grecia, Polonia, Italia, Corea, China, Singapur, Irán y Arabia Saudita. Uno de los estudios es multicéntrico en el continente europeo. El $42 \%$ de los estudios proceden de los Estados Unidos. Dichos artículos son producto de investigaciones desarrolladas por enfermería, medicina y grupos multidisciplinarios, teniendo como metodología estudios descriptivos observacionales, prospectivo longitudinal y correlacionales de corte transversal. Se utilizaron como muestra pacientes con IC atendidos en el ámbito ambulatorio y hospitalario, incluyendo pacientes mayores de edad con fracción de eyección del ventrículo izquierdo (FEVI) preservada y deprimida. Solo en un estudio se evaluó la diada paciente-familiar. Se identificaron 24 factores deteriorantes, los cuales se clasificaron en las dimensiones física, social y psicológica del individuo para facilitar su análisis.

\section{Dimensión física}

Hace referencia al estado de la salud física de la persona con IC y está determinada por diferentes factores, los cuales se dividen en tres grupos:

\section{Características del individuo}

Hay condiciones propias del individuo que aumentan la posibilidad de que la IC genere mayor deterioro de la CVRS. Uno de los factores es la edad. En algunos de los estudios incluidos en la revisión se concluye que los pacientes más jóvenes presentan un mayor efecto negativo de la IC y por tanto una peor CVRS $^{13-21}$. Sin embargo, otros estudios realizados con pacientes españoles y saudís contradicen este hallazgo, ${ }^{4,22}$. Por su parte, Rahnavard et al18 no encontraron una relación estadísticamente significativa entre la edad y la CVRS en pacientes mujeres iraníes. 
Tabla 1. Artículos seleccionados.

\begin{tabular}{|c|c|c|}
\hline ESTUDIO & FACTORES & RESULTADOS \\
\hline $\begin{array}{l}\text { Comín-Colet } \\
\text { et }\left.a\right|^{4}\end{array}$ & Sociodemográficos/clínicos & $\begin{array}{l}\text { Ina mayor edad, sexo femenino, peor CF, mayor comorbilidad y el ingreso } \\
\text { eciente por IC predicen una peor CVRS. }\end{array}$ \\
\hline Chu et $a 1^{13}$ & Sociodemográficos/clínicos & $\begin{array}{l}\text { El menor ingreso familiar total, peor CF, sexo femenino y edad más joven se } \\
\text { asocian con una peor CVRS. }\end{array}$ \\
\hline $\begin{array}{l}\text { Gallagher et } \\
a^{14}\end{array}$ & $\begin{array}{l}\text { Deterioro cognitivo - Apoyo } \\
\text { social }\end{array}$ & $\begin{array}{l}\text { El bajo nivel de apoyo social, edad más joven y el estado cognitivo se asocian } \\
\text { con el deterioro de la CVRS. }\end{array}$ \\
\hline Heo et $a 1^{15}$ & $\begin{array}{l}\text { Apoyo social, síntomas } \\
\text { físicos y depresivos }\end{array}$ & $\begin{array}{l}\text { El apoyo social no se asoció con la CVRS, sin embargo, los pacientes con mayor } \\
\text { apoyo emocional tenían síntomas físicos y depresivos menos graves, lo que se } \\
\text { relaciona con mejor CVRS. }\end{array}$ \\
\hline Hwang et $a l^{16}$ & $\begin{array}{l}\text { Sociodemográficos, clínicos } \\
\text { y depresión }\end{array}$ & $\begin{array}{l}\text { La edad más joven, la duración de la IC, la presencia de síntomas físicos y la } \\
\text { depresión deterioran la CVRS. }\end{array}$ \\
\hline Nesbitt et $a l^{17}$ & $\begin{array}{l}\text { Sociodemográficos, clínicos } \\
\text { y la depresión - ansiedad }\end{array}$ & $\begin{array}{l}\text { La edad más joven, sexo masculino, peor CF, déficit de conocimiento de la IC, } \\
\text { peor control percibido y la presencia de síntomas de depresión-ansiedad } \\
\text { afectan la CVRS. }\end{array}$ \\
\hline $\begin{array}{l}\text { Rahnavard et } \\
\left.a\right|^{18}\end{array}$ & Sociodemográficos/clínicos & $\begin{array}{l}\text { peor CF, la duración de la enfermedad, rehospitalizaciones y nivel educativo } \\
\text { o se relacionan con una peor CVRS. }\end{array}$ \\
\hline Stamp et $a l^{19}$ & Sociodemográficos/clínicos & $\begin{array}{l}\text { y el funcionamiento familiar bajo presentan } \\
\text { onal. }\end{array}$ \\
\hline Wu et & & \\
\hline Sousa et $a^{21}$ & Sociodemográficos/clínicos & $\begin{array}{l}\text { CVRS y a mayor edad mejora la percepción y } \\
\text { lad. }\end{array}$ \\
\hline AbuRuz et $a 1^{22}$ & Clínicos - Apoyo social & $\begin{array}{l}\text { eprimida, mayor edad y menor soporte tangible se relacionan con } \\
\text { S }\end{array}$ \\
\hline $\begin{array}{l}\text { Aggelopoulou } \\
\text { et } a l^{23}\end{array}$ & Ansiedad y depresión & $\begin{array}{l}\text { siedad y depresión se asocian con peor CVRS. La edad, la duración de } \\
\text { olifarmacia se relacionaron con la CVRS. }\end{array}$ \\
\hline $\begin{array}{l}\text { Gathright et } \\
a l^{24}\end{array}$ & Deterioro cognitivo & asocian con peor CVRS. No hay \\
\hline Faxén et $a l^{25}$ & & cientes con FEVI preservada. \\
\hline Loo et $a^{26}$ & $\begin{array}{l}\text { Sociodemográficos } \\
\text { depresión }\end{array}$ & $\begin{array}{l}\text { ejor CVRS. Niveles más bajos de autoeficacia y la } \\
\text { CVRS. }\end{array}$ \\
\hline $\begin{array}{l}\text { Uchmanowicz } \\
\text { et }\left.a\right|^{27}\end{array}$ & Fragilidad & $\begin{array}{l}\text { acto negativo en la CVRS en pacientes ancianos. } \\
n \text { se relacionan con peor CVRS. }\end{array}$ \\
\hline $\begin{array}{l}\text { Banerjee et } \\
\left.a\right|^{28}\end{array}$ & Control percibido & $\begin{array}{l}\text { trol percibido se asocia con menos síntomas depresivos, } \\
\text { r CVRS. }\end{array}$ \\
\hline Heo et $a l^{29}$ & $\begin{array}{l}\text { Clínicos, psicosociales y de } \\
\text { comportamiento }\end{array}$ & $\begin{array}{l}\text { Una peor CF, la presencia de síntomas depresivos y un menor control percibido } \\
\text { se asocian con peor CVRS. }\end{array}$ \\
\hline Lee et $a \beta^{32}$ & La actividad física & Un estilo de vida físicamente inactivo y una peor CF se relaciona con peor CVRS. \\
\hline Heo et $a l^{\beta 1}$ & Control percibido & $\begin{array}{l}\text { Mayor control percibido, menos síntomas depresivos y mejor CF se relacionan } \\
\text { con mejor CVRS. }\end{array}$ \\
\hline Patron et $a \beta^{34}$ & $\begin{array}{l}\text { Síntomas somático-afectivo } \\
\text { vs cognitivo-depresivo }\end{array}$ & $\begin{array}{l}\text { Los síntomas somático-afectivos más graves de la depresión se asociaron con } \\
\text { peor CVRS. }\end{array}$ \\
\hline Bhatt et $\left.a\right|^{35}$ & Depresión & Una mayor depresión se asoció con peor CVRS. \\
\hline Moryś et $a \beta^{36}$ & $\begin{array}{l}\text { Depresión } \\
\text { cognitivo }\end{array}$ & $\begin{array}{l}\text { Una mayor depresión se asoció con peor CVRS. No se identificó relación entre } \\
\text { la CVRS y el deterioro cognitivo. }\end{array}$ \\
\hline Lee et $a 1^{37}$ & Síntomas depresivos & $\begin{array}{l}\text { La depresión media la relación entre la CVRS y la supervivencia libre de eventos } \\
\text { cardíacos. }\end{array}$ \\
\hline
\end{tabular}

*CVRS: Calidad de Vida Relacionada con la Salud. *IC: Insuficiencia Cardiaca. *CF: Capacidad Funcional. 
En el factor sexo no se encontraron diferencias estadísticamente significativas entre hombres y mujeres en la CVRS, alterándose en igual medida, independientemente de la edad y la gravedad de la enfermedad ${ }^{23-25}$. Pero la CVRS pobre puede ser multifactorial y compleja en las mujeres. Varios autores ${ }^{4,13,21,26}$ coinciden en que el sexo femenino tiene una peor CVRS. Incluso Comín et $a l^{4}$ encontraron que esta relación podría estar relacionada con la pérdida de rol social de la mujer. Por el contrario, Nesbitt et $\left.a\right|^{17}$ identificaron una peor CVRS en los hombres, en población rural.

El estudio de Uchmanowicz y Gobbens ${ }^{27}$ evaluó la fragilidad usando la escala del Indicador de Fragilidad de Tilburg (TFI), el cual consta de características sociodemográficas, posibles determinantes de la fragilidad y los componentes de la fragilidad, divididos en los dominios físico, psicológico y social. Identificaron que el aumento en la fragilidad se relaciona con el deterioro de la CVRS. Adicionalmente, la presencia de condiciones comórbidas aumenta la complejidad de la atención al paciente, ya que repercute en un mayor deterioro de la condición física y psicosocial, y es un factor influyente para el deterioro de la CVRS $4,13,15,19,28$.

\section{Estado de la insuficiencia cardiaca}

Los factores asociados al diagnóstico de la IC se han identificado no solo como puntos diana en el planteamiento de estrategias de intervención terapéutica, sino también por su importancia al contribuir al deterioro de la CVRS de la persona con IC. Dentro de este grupo de factores se encuentra la FEVI. AbuRuz et $a l^{22}$ encontraron en su estudio que los pacientes saudís con FEVI deprimida tenían una peor calidad de vida.

La presencia de síntomas físicos se asocia también a una peor $\mathrm{CVRS}^{16,18,29}$. Heo et al ${ }^{29}$ identificaron que los síntomas físicos se relacionan con la presencia de síntomas depresivos y con la ingesta de sodio, y median la relación entre los síntomas depresivos y la CVRS.

La Capacidad Funcional (CF), la cual hace referencia a la capacidad que tiene la persona para la realización de actividades de la vida diaria que requieren esfuerzo físico ${ }^{30}$, empeora por la presencia de síntomas físicos de la IC, siendo un factor deteriorante de la CVRS $4,13,15,17,18,23,24,28,29,31,32$. Según la clasificación NYHA, los pacientes con clase funcional II/IV presentaron menos síntomas de ansiedad y depresión y mejor CVRS en comparación con los pacientes con clases funcionales III-IV/IV ${ }^{23,28}$. Wu et $a l^{20}$ la midieron a través del instrumento DASI y encontraron que una peor CF está asociada significativamente con una mayor carga de comorbilidad y una peor CVRS.

La duración y la gravedad de la IC, que pueden estar relacionadas con la presencia de síntomas físicos, el deterioro de la CF y la refractariedad al tratamiento, también deterioran la $\mathrm{CVRS}^{2,4,16,18}$. Incluso, Aggelopoulou et $a l^{23}$ encontraron en su estudio que influye en la presencia de síntomas de ansiedad y depresión. A su vez, los ingresos hospitalarios, un factor estrechamente relacionado con los anteriormente mencionados, y la polifarmacia también empeoran la $\mathrm{CVRS}^{4,18}$. También se observó una fuerte correlación entre la CVRS, la edad, la duración de la IC, la duración del régimen de medicación, el número de medicaciones y las dosis diarias $^{23}$.

\section{Factores conductuales}

Lee et $\mathrm{a}^{32}$ identificaron que la CVRS se correlacionó positivamente con la autoeficacia del ejercicio mientras que con un estilo de vida físicamente inactivo y una peor $\mathrm{CF}$, según la NYHA, mostró una relación significativamente negativa.

\section{La dimensión social}

Hace referencia al funcionamiento social del individuo determinado por diferentes factores que se dividen en tres grupos:

\section{Características sociales del individuo}

El nivel educativo fue un factor identificado por varios estudios, determinando que a mayor nivel educativo mejor salud física y emocional, y por ende mejor $\operatorname{CVRS}^{18,20,23,26,32}$, relación que no fue 
identificada por Gathright et $a l^{24}$. El estado civil se asocia con la $\mathrm{CVRS}^{15}$ y tiene un efecto significativo sobre los síntomas de depresión y ansiedad ${ }^{23,28}$. Los pacientes que viven con su pareja o con otras personas tienen mayor apoyo psicológico y emocional y síntomas físicos menos graves. Por el contrario, no hubo relación estadísticamente significativa entre el estado civil y la CVRS en pacientes mujeres iraníes ${ }^{18}$. El estado laboral también fue analizado y algunos estudios encontraron que las personas empleadas tienen mejor CVRS en comparación con aquellas dedicadas al hogar, que están jubiladas o desempleadas ${ }^{23,32}$. A este factor se asocia el estado económico, determinado por una buena situación financiera, el cual influye positivamente en la CVRS $13,23,32$.

\section{Apoyo social}

Hace referencia a la percepción que tiene la persona del apoyo que recibe de su familia, amigos y comunidad. El apoyo social se asocia con los síntomas físicos y depresivos y, a través de ellos, con la CVRS15, lo que permite determinar que a mayor apoyo social menor efecto negativo de la IC en la CVRS de la persona ${ }^{14}$. AbuRuz et $\left.a\right|^{22}$ evaluaron el apoyo social a través de la Encuesta de Apoyo Social del Estudio de Resultados Médicos (MOS-SS), la cual establece cuatro dominios del apoyo social: apoyo emocional/informativo, apoyo tangible, interacción social positiva y afecto $^{33}$. Encontraron que el apoyo tangible o instrumental, el cual hace referencia a las conductas para la solución de problemas, mejora la CVRS de las personas con IC.

Por otra parte, entendiendo el apoyo emocional como el apoyo intangible de otras personas, Heo et $a l^{15}$ identificaron en su estudio que los pacientes con mayor apoyo emocional tenían síntomas depresivos menos graves. Este tipo de apoyo estuvo significativamente relacionado con los síntomas físicos, los síntomas depresivos y, finalmente, con la CVRS.

\section{Apoyo familiar}

Stamp et $a l^{19}$ determinaron en su estudio que el funcionamiento familiar y la crítica percibida muestran una asociación significativa con los síntomas depresivos y la dimensión emocional de la CVRS en los pacientes con IC. Adicionalmente, evidenciaron que la etnia puede afectar los síntomas depresivos, pues, al evaluar una muestra de pacientes estadunidenses, identificaron que los afroamericanos, a diferencia de las personas de raza blanca, pueden tener mayor capacidad de recuperación y otras formas de apoyo social cuando el funcionamiento familiar es bajo. Concluyen que el funcionamiento familiar, el apoyo a la autonomía y el conocimiento de la IC de los familiares pueden ser influencias importantes en los niveles de síntomas depresivos y el aspecto emocional de la CVRS.

\section{La dimensión psicológica}

Hace referencia a la salud mental y emocional del individuo, que, al no presentar alteraciones, contribuye positivamente a la CVRS de las personas con $\mathrm{IC}^{22}$. Está conformada por diferentes factores.

\section{La ansiedad y la depresión}

Aggelopoulou et $\left.a\right|^{23}$ y Banerjee et $\left.a\right|^{28}$ encontraron en sus estudios que los pacientes con IC experimentan altos niveles de ansiedad y depresión, que, asociado a factores socioeconómicos como la edad, el sexo, el estado civil y el nivel educativo, afectan negativamente la CVRS general y en sus dominios físico y mental. Por su parte, en el estudio de Patron et $a l^{34}$ no se mostró relación de la ansiedad con el deterioro de la CVRS.

De manera independiente, la depresión se asocia de forma negativa significativamente con la CVRS, incluso desde la presencia de síntomas leves, y así lo demuestran los resultados de diversos estudios incluidos en esta revisión ${ }^{15-17,24,26,27,29,31,35,36}$. Los síntomas depresivos moderados a severos se asociaron al $70 \%$ más de ingresos hospitalarios por todas las causas y 2,5 veces más de admisiones relacionadas con la IC ${ }^{35}$. También median la relación entre la CVRS y la supervivencia libre de eventos cardiacos, de acuerdo con lo identificado por Lee et $a b^{37}$. La depresión incluye síntomas cognitivodepresivos (sentimientos de culpa, autoimagen negativa, entre otros) y síntomas somático-afectivos 
(problemas de sueño, fatiga y pérdida de energía, entre otros). Según Patron et $a l^{34}$, los síntomas somático-afectivos se relacionaron con el deterioro de la CVRS, mientras que los síntomas cognitivodepresivos no mostraron relación. Stamp et al ${ }^{19}$ identificaron que la etnia puede afectar los síntomas depresivos y determinaron que las personas afroamericanas tienen características específicas y formas de apoyo social que contribuyen a tener menos síntomas de este tipo. Siendo la fragilidad un factor deteriorante de la CVRS, Uchmanowicz y Gobbens $^{27}$ identificaron en su estudio correlaciones positivas significativas entre la fragilidad, la ansiedad y la depresión: a mayor nivel de fragilidad mayor nivel de ansiedad y depresión.
Gallagher et $a l^{14}$ partieron, para el desarrollo de su estudio, de hallazgos en la literatura científica en los cuales se establece que "el deterioro cognitivo es prevalente en los pacientes con IC y se asocia con peores resultados" y "el deterioro asociado con la IC tiene un efecto negativo en varias áreas, especialmente la memoria operativa, la memoria, la función ejecutiva, el autocuidado y las actividades de la vida diaria". En sus resultados encontraron que el estado cognitivo predijo tanto el dominio físico como la CVRS en general. Por su parte, Gathright et $a l^{24} y$ Morys et $a l^{36}$ identificaron que no hay relación entre la CVRS y los dominios de la función cognitiva.

\section{Deterioro cognitivo}

Tabla 2. Factores que deterioran la CVRS de las personas con IC.

\begin{tabular}{|c|c|c|}
\hline \multirow{9}{*}{ 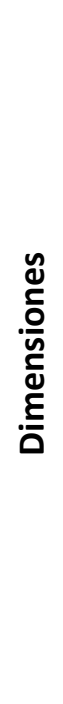 } & \multirow{3}{*}{ 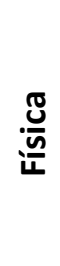 } & Características del individuo: edad joven, sexo, fragilidad y comorbilidad. \\
\hline & & $\begin{array}{l}\text { Estado de la IC: FEVI deprimida, síntomas físicos, capacidad funcional, duración y gravedad } \\
\qquad \text { de la IC, ingresos hospitalarios y polifarmacia. }\end{array}$ \\
\hline & & $\begin{array}{l}\text { Factores conductuales: estilo de vida físicamente inactivo y menor autoeficacia del } \\
\text { ejercicio }\end{array}$ \\
\hline & \multirow{3}{*}{$\overline{\frac{\pi}{\pi}}$} & $\begin{array}{l}\text { Características sociales del individuo: menor nivel educativo, estado civil (sin pareja), } \\
\text { estado laboral (desempleado, jubilado) y peor estado económico. }\end{array}$ \\
\hline & & Apoyo social: menor apoyo tangible y apoyo emocional \\
\hline & & Apoyo familiar: funcionamiento familiar, crítica percibida y apoyo a la autonomía \\
\hline & \multirow{3}{*}{$\begin{array}{l}\frac{\pi}{80} \\
\frac{0}{0} \\
\frac{0}{0} \\
0\end{array}$} & Ansiedad \\
\hline & & Depresión \\
\hline & & Deterioro cognitivo \\
\hline
\end{tabular}

\section{Control percibido}

Definido como "la creencia de que uno tiene los recursos necesarios para enfrentar los eventos negativos y la capacidad de influir positivamente en las consecuencias de esos eventos negativos" ${ }^{28}$, es un factor que tiene una relación significativa con la $\mathrm{CVRS}^{17,29,31}$. Banerjee et $a l^{28}$ evaluaron la relación existente entre el control percibido y la CVRS, y encontraron que se correlacionan negativamente, de forma significativa, aunque débil. También tuvo una relación inversa con la ansiedad y la depresión.
Determinaron que el estado psicológico puede tener un posible efecto mediador sobre la asociación entre el control percibido y la CVRS. Las actitudes más positivas y menos barreras para seguir una dieta baja en sodio y más apoyo social se asociaron significativamente con niveles más altos de control percibido ${ }^{31}$. En la tabla 2 se resumen los 24 factores descritos previamente.

\section{DISCUSIÓN}

El interés en el estudio de la IC, en particular, 
obedece en gran medida a que las investigaciones reportadas por la literatura científica han demostrado que las personas con esta enfermedad tienen un deterioro significativo de su CVRS en comparación con personas que padecen otro tipo de enfermedades crónicas. Tanto en América Latina, como en el resto del mundo, la IC es una enfermedad de gran impacto para la persona y para los sistemas de salud, a la que se suman etiologías propias de la región ${ }^{38}$, lo que implica un abordaje especial por las dificultades de acceso a los servicios de salud $y$ al tratamiento oportuno. Pese a su importancia, en la presente revisión solo se identificó un estudio realizado en Latinoamérica ${ }^{21}$, lo que justifica la realización de un mayor número de investigaciones en este tipo de población, que permita hacer un comparativo con los hallazgos en otros continentes.

El total de los artículos seleccionados son observacionales. Esto puede generar un sesgo en esta revisión por su enfoque integrativo. Es posible que se disponga de investigaciones experimentales, así como de abordaje cualitativo, cuyos aportes enriquecen el conocimiento disciplinar y deben ser contemplados en futuras revisiones sobre esta temática.

En relación con la dimensión física, los factores concernientes a la edad y el sexo deben ser tenidos en cuenta en el momento de diseñar intervenciones que se ajusten a las características y necesidades individuales. Aunque no hay evidencia científica significativa de que las mujeres presentan peor CVRS en comparación con el sexo masculino, es necesario que las acciones en salud estén orientadas a la complejidad que las caracteriza, pues el deterioro de la CVRS es multifactorial. Las mujeres pueden llegar a desconocer la importancia de la enfermedad cardiovascular, adicional a la percepción y el manejo específico de su salud que resulta de sus patrones de crianza, su herencia sociocultural, su nivel educativo y el rol que desempeñan a nivel familiar y social ${ }^{39}$.

El otro aspecto esencial para tener en cuenta en la dimensión física es la fragilidad. Como fenotipo, la fragilidad es un síndrome clínico en el que están presentes tres o más de los siguientes criterios: pérdida de peso involuntaria, agotamiento, debilidad, velocidad lenta para caminar y bajo nivel de actividad física. Se asocia a condiciones como ser afroamericano, tener educación e ingresos más bajos, peor salud y tasas más altas de enfermedades crónicas comórbidas y discapacidad, siendo esta última el resultado de la fragilidad $^{40}$. Como indicador, a través del índice de fragilidad se puede medir el grado de vulnerabilidad y la edad biológica de una persona, estableciendo la relación entre el número de déficits presentes y el número total de déficits considerados; la fragilidad es la acumulación de déficits que afectan las reservas y la capacidad de responder a factores estresantes ${ }^{41}$.

La fragilidad es un aspecto importante en la valoración del adulto mayor con enfermedad cardiovascular y su evaluación permite refinar las estimaciones de riesgo y guiar planes de tratamiento personalizados, facilitando así el logro de resultados positivos en morbimortalidad, pues la fragilidad puede predisponer a la persona con IC a descompensaciones y hospitalizaciones más frecuentes, aumentando la mortalidad y deteriorando la $\mathrm{CVRS}^{42,43}$.

En lo referente al estado clínico de la IC, se resalta la CF como factor deteriorante de la CVRS. La reducción de la CF se asocia con cambios del estado funcional. Las personas con una insuficiencia orgánica, tal como la IC, tienen una trayectoria de deterioro funcional específica que se caracteriza por su fluctuación individual ajustada al patrón clínico de exacerbaciones de la enfermedad ${ }^{44}$. Este comportamiento clínico se debe tener cuenta para la prestación de servicios de salud con un enfoque clínico diferente. Las fluctuaciones en la estabilidad de la enfermedad y la presencia de descompensaciones se asocian, en gran porcentaje, con la baja adherencia del paciente al tratamiento farmacológico, la restricción en el consumo de líquidos y sodio, y al cumplimiento de hábitos de vida saludable. Auld et $a l^{45}$ encontraron que el empeoramiento de la CVRS en su dimensión física se asoció con un mantenimiento deficiente del autocuidado. Es así como el empoderamiento del paciente en el reconocimiento de los síntomas de descompensación de la IC y el fortalecimiento del autocuidado permiten disminuir la tasa de 
rehospitalizaciones y el consecuente deterioro en la $\mathrm{CVRS}^{46-48}$.

La autoeficacia, entendida como "la creencia de uno en su capacidad para promover y mantener una buena salud" 49 , se considera también un predictor de CVRS en estos pacientes ${ }^{26,31}$ y es importante en el proceso de mantenimiento del autocuidado. La adaptación de intervenciones dirigidas a mejorar la autoeficacia también puede ser clave para impactar en la hospitalización y en la $\mathrm{CVRS}^{45},{ }^{50}$ En este proceso, la aceptación de la enfermedad contribuye al logro de los objetivos de tratamiento, pues el paciente que la acepta participa activamente en el proceso terapéutico, lo que contribuye a mejorar su $\mathrm{CVRS}^{49}$.

Por su parte, los factores sociales identificados deben contemplarse al momento de establecer el tratamiento, pues niveles educativos bajos pueden afectar la comprensión de la enfermedad y el cumplimiento de las pautas dadas por los profesionales de la salud. Una valoración multidisciplinaria facilita la identificación de estos factores para la definición de acciones encaminadas a su fortalecimiento, lo que repercute positivamente en la presencia de síntomas físicos y depresivos, más aún cuando la evidencia en la literatura reafirma el impacto negativo que tiene la presencia de síntomas depresivos sobre la CVRS de la persona con IC.

Finalmente, el control percibido influye en la capacidad que tiene el individuo para afrontar los cambios en su condición de salud y se relaciona significativamente con la CVRS. Su significado es asemeja al de resiliencia, entendiéndola como la habilidad que tiene un individuo para ajustarse a las adversidades, mantener el equilibrio y continuar viviendo de una manera positiva. La persona resiliente posee mayor habilidad para atenuar la presión causada por el impacto negativo de la enfermedad ${ }^{51,52}$. Cuando existe la presencia de síntomas de ansiedad y depresión, la resiliencia disminuye $\mathrm{fl}^{51,53}$; por lo tanto, se hace necesario implementar acciones orientadas a promover $y$ mantener la resiliencia, para mejorar la capacidad de la persona de enfrentar la IC, desarrollando habilidades tales como una respuesta rápida ante el riesgo, capacidad de autogestión, búsqueda de información, relaciones interpersonales positivas, optimismo y asunción de responsabilidad ${ }^{52}$.

\section{CONCLUSIÓN}

La determinación de la CVRS de la persona con IC requiere que los profesionales de la salud realicen una evaluación integral del individuo para poder definir aquellos factores que influyen en su deterioro, los cuales deben ser contemplados en el diseño e implementación de intervenciones específicas, con un componente educativo que fortalezca las conductas de autocuidado y mejore la CVRS, contribuyendo finalmente a un mejor uso de los servicios de salud, optimizando los recursos para el logro de los objetivos de atención.

La presente revisión no encontró estudios que evalúen la CVRS en la población colombiana con IC; por lo tanto, es necesario que se desarrollen investigaciones que permitan conocer este fenómeno en el contexto particular. Dicha información permitirá establecer similitudes y diferencias con los resultados en otros puntos geográficos del mundo, y contribuirá a fortalecer el cuerpo de conocimientos disciplinar $y$ al establecimiento de guías de manejo para la población.

\section{DECLARACIÓN SOBRE CONFLICTO DE INTERESES}

Los autores declaran no tener conflicto de intereses.

\section{CONTRIBUCIÓN DE LOS AUTORES}

Primer autor: búsqueda, revisión de los artículos, resultados y discusión.

Segundo autor: discusión.

\section{REFERENCIAS BIBLIOGRÁFICAS}

1. Jaramillo C, Gómez L EA, Hernández E, Saldarriaga

C, Florez N, Buitrago R, et al. Consenso colombiano para el diagnóstico y tratamiento de la insuficiencia 
cardiaca crónica. Soc Colomb Cardiol y cirugía Cardiovasc. 2014.2 Disponible en: http://scc.org.co/wpcontent/uploads/2015/01/cronica-completaweb.pdf

2. Ponikowski $P$, Voors $A A$, Anker SD, Bueno $H$, Cleland JGF, Coats AJS, et al. ESC Guidelines for the Diagnosis and Treatment of Acute and Chronic Heart Failure. Rev Española Cardiol [Internet]. 2016 Dec;69(12):1167. Disponible en: http://eurheartj.oxfordjournals.org/content/ehj/ea rly/2016/05/19/eurhe artj.ehw128.full.pdf

3. Gómez L EA. Introducción, epidemiología de la falla cardiaca e historia de las clínicas de falla cardiaca en Colombia. Clínicas de falla cardiaca. Rev Col Cardiol. 2016;23 Supl 1:1:6-12. Doi: http://dx.doi.org/10.1016/j.rccar.2016.01.004

4. Comín-Colet J, Anguita M, Formiga F, Almenar L, Crespo-Leiro MG, Manzano L, et al. Calidad de vida relacionada con la salud de los pacientes con insuficiencia cardiaca crónica sistólica en España: resultados del estudio VIDA-IC. Rev Española Cardiol [Internet]. 2016;69(3):256-71. Doi: http://dx.doi.org/10.1016/j.recesp.2015.07.034

5. Etxeberría-Lekuona D, Sánchez-Alvarez J, Alonso Gutiérrez A, Acha Arrieta V, Campos Rivas R, Jarne Betrán V. Estudio de la calidad de vida de pacientes con insuficiencia cardiaca en un Servicio de Medicina Interna. An Med Interna [Internet]. 2007;24. Disponible en: http://pesquisa.bvsalud.org/portal/resource/es/m dl-17590089

6. Achury Diana Marcela. Autocuidado y adherencia en pacientes con falla cardiaca. Aquichan. 2007;7(2):139-60. Disponible en: http://aquichan.unisabana.edu.co/index.php/aquic han/article/view/109/219

7. Lugo A LH, García G HI, Gómez RC. Confiabilidad del cuestionario de calidad de vida en salud sf-36 en Medellín, Colombia. Colomb Rev Fac Nac Salud Pública [Internet]. 2006;24(2):37-50. Available from:

http://www.redalyc.org/articulo.oa?id=12024205
8. Urzúa MA. Calidad de vida relacionada con la salud: elementos conceptuales. Rev Med Chil [Internet]. $2010 \quad$ Mar;138(3):358-65. Doi: http://dx.doi.org/10.4067/S003498872010000300017

9. Bredow T, Peterson S. Health - Related Quality of life. In: Middle range theories: application to nursing research. Philadelphia: Lippincott Williams \& Wilkins; 2004.

10. Botero BE, Pico ME. Calidad de vida relacionada con la salud (CVRS) en adultos mayores de 60 años: una aproximación teórica. Hacia la Promoción la salud [Internet]. 2007;12:11-24. Disponible en: http://www.scielo.org.co/pdf/hpsal/v12n1/v12n1a 01.pdf

11. Crossetti, MGO. Revisão integrativa de pesquisa na enfermagem o rigor cientifico que lhe é exigido [editorial]. Rev Gaúcha Enferm., Porto Alegre (RS) 2012 jun;33(2):10-11. Doi: http://dx.doi.org/10.1590/S198314472012000200002

12. Von Elm $E$, Altman $D$, Egger $M$, Pocock $S$, Gøtzsche P, Vandenbroucke J. Declaración de la Iniciativa STROBE (Strengthening the Reporting of Observational Studies in Epidemiology): directrices para la comunicación de estudios observacionales. Rev Esp Salud Pública. 2008;82:251-9. Disponible en:

http://scielo.isciii.es/scielo.php?script=sci_arttext\& pid=S1135-57272008000300002

13. Chu SH, Lee WH, Yoo JS, Kim SS, Ko IS, Oh EG, Lee J, Choi M, Cheon JY, Shim CY, Kang SM. Factors affecting quality of life in Korean patients with chronic heart failure. Japan J Nurs Sci. 2014;11(1):54-64. Doi: https://doi.org/10.1111/jjns.12002

14. Gallagher R, Sullivan A, Burke R, Hales S, Sharpe PTG. Quality of life, social support and cognitive impairment in heart failure patients without diagnosed dementia. Int J Nurs Pr. 2016;22(2):17988. Doi: https://doi.org/10.1111/ijn.12402

15. Heo S, Lennie TA, Moser DK, Kennedy RL. Types of social support and their relationships to physical 
and depressive symptoms and health-related quality of life in patients with heart failure. Hear Lung J Acute Crit Care. 2014;43:299-305. Doi: https://doi.org/10.1016/j.hrtlng.2014.04.015

16. Hwang SL, Liao WC, Huang TY. Predictors of quality of life in patients with heart failure. Jpn J Nurs Sci. 2014;11(4):290-298. Doi: https://doi.org/10.1111/jjns.12034

17. Nesbitt T, Doctorvaladan S, Southard JA, Singh S, Fekete A, Marie K, et al. Correlates of Quality of Life in Rural Patients With Heart Failure. Circ Hear Fail. $2014 \quad$ Nov 1;7(6):882-7. Doi: https://doi.org/10.1161/CIRCHEARTFAILURE.113.0 00577

18. Rahnavard Z, Nodeh ZH, Hatamipour K. Congestive heart failure: Predictors of healthrelated quality of life in Iranian women. Contemp Nurse. 2014;47(1-2):159-67. Doi: https://doi.org/10.5172/conu.2014.47.1-2.159

19. Stamp KD, Dunbar SB, Clark PC, Reilly CM, Gary RA, Higgins $M$, et al. Family context influences psychological outcomes of depressive symptoms and emotional quality of life in patients with heart failure. J Cardiovasc Nurs. 2014;29(6):517-527. Doi: https://doi.org/10.1097/JCN.0000000000000097

20. Wu JR, Lennie TA, Frazier SK, Moser DK. HealthRelated Quality of Life, Functional Status, and Cardiac Event-Free Survival in Patients With Heart Failure. J Cardiovasc Nurs. 2016;31(3):236-244. Doi: https://doi.org/10.1097/JCN.0000000000000248

21. Sousa MM de, Oliveira J dos S, Soares MJGO, Bezerra SMM da S, Araújo AA de, Oliveira SH dos $S$, et al. Association of social and clinical conditions to the quality of life of patients with heart failure. Rev Gaúcha Enferm. 2017;38(2). Doi: https://doi.org/10.1590/1983-1447.2017.02.65885

22. AbuRuz ME, Alaloul F, Saifan A, Masa'deh R, Abusalem S. Quality of Life for Saudi Patients With Heart Failure: A Cross-Sectional Correlational Study. Glob J Health Sci. 2016 Jun 25;8(3):49-58. Doi: https://doi.org/10.5539/gjhs.v8n3p49
23. Aggelopoulou Z, Fotos N V, Chatziefstratiou AA, Giakoumidakis K, Elefsiniotis I, Brokalaki H. The level of anxiety, depression and quality of life among patients with heart failure in Greece. Appl Nurs Res. 2017 Apr 1;34:52-6. Doi: https://doi.org/10.1016/j.apnr.2017.01.003

24. Gathright EC, Fulcher MJ, Dolansky MA, Gunstad $J$, Redle JD, Josephson R, et al. Cognitive Function Does Not Impact Self-reported Health-Related Quality of Life in Heart Failure Patients. J Cardiovasc Nurs. 2016;31(5):405-11. Doi: https://doi.org/10.1097/JCN.0000000000000277

25. Faxén UL, Hage C, Donal E, Daubert J-C, Linde C, Lund LH. Patient reported outcome in HFpEF: Sexspecific differences in quality of life and association with outcome. Int J Cardiol. 2018;267:128-32. Doi: https://doi.org/10.1016/j.ijcard.2018.04.102

26. Loo DWY, Jiang Y, Koh KWL, Lim FP, Wang W. Self-efficacy and depression predicting the healthrelated quality of life of outpatients with chronic heart failure in Singapore. Appl Nurs Res. 2016 Nov 1;32:148-55.

Doi:

https://doi.org/10.1016/j.apnr.2016.07.007

27. Uchmanowicz I, Gobbens RJ. The relationship between frailty, anxiety and depression, and healthrelated quality of life in elderly patients with heart failure. Clin Interv Aging. 2015 Oct 5;10:1595. Doi: https://doi.org/10.2147/CIA.S90077

28. Banerjee T, Lee KS, Browning SR, Hopenhayn C, Westneat $\mathrm{S}$, Biddle $\mathrm{MJ}$, et al. Limited association between perceived control and health-related quality of life in patients with heart failure. J Cardiovasc Nurs. 2014;29(3):227-31. Doi: https://doi.org/10.1097/JCN.0b013e31828b2b23

29. Heo S, Moser DK, Lennie TA, Fischer M, Smith E, Walsh MN. Modifiable correlates of physical symptoms and health-related quality of life in patients with heart failure: A cross-sectional study. Int J Nurs Stud. 2014; Doi: https://doi.org/10.1016/j.ijnurstu.2014.03.005

30. Sánchez EM, Vera CM, Navas C, Ortiz SD, Rodríguez C, Vargas DM, et al. Validación para 
Colombia del cuestionario para la "Medición de la capacidad funcional en pacientes con falla cardíaca" Rev Col Cardiol. 2018;25(6):356-65. Doi: https://doi.org/10.1016/j.rccar.2018.04.004

31. Heo S, Lennie TA, Pressler SJ, Dunbar SB, Chung $M L M D$. Factors associated with perceived control and the relationship to quality of life in patients with heart failure. Eur J Cardiovasc Nurs. 2015;14(2):13744.

Doi: https://doi.org/10.1177/1474515113519931

32. Lee $H$, Boo $S, Y u J$, Suh $S$, Chun KJ, Kim JH. Physical Functioning, Physical Activity, Exercise Selfefficacy, and Quality of Life Among Individuals With Chronic Heart Failure in Korea: A Cross-sectional Descriptive Study. J Nurs Res. 2017 Apr 1;25(2):1319. https://doi.org/10.1097/JNR.0000000000000150

33. Londoño A NH, Rogers HL, Castilla T JF, Posada G SL, Ochoa A NL, Jaramillo P MA, et al. Validación en Colombia del cuestionario MOS de apoyo social. Int J Psychol Res. 2012;5(1):142-50. Disponible en: http://mvint.usbmed.edu.co:8002/ojs/index.php/w eb

34. Patron E, Messerotti Benvenuti S, Lopriore V, Aratari J, Palomba D. Somatic-Affective, But Not Cognitive-Depressive Symptoms are Associated With Reduced Health-Related Quality of Life in Patients With Congestive Heart Failure. Psychosomatics. 2017;58:281-91. Doi: https://doi.org/10.1016/j.psym.2017.01.001

35. Bhatt KN, Kalogeropoulos AP, Dunbar SB, Butler J, Georgiopoulou V V. Depression in heart failure: Can PHQ-9 help? Int J Cardiol. 2016 Oct 15;221:24650.

Doi:

https://doi.org/10.1016/j.ijcard.2016.07.057

36. Moryś JM, Pąchalska M, Bellwon J, Gruchała M. Cognitive impairment, symptoms of depression, and health-related quality of life in patients with severe stable heart failure. Int J Clin Heal Psychol. 2016;16:230-8.

Doi: https://doi.org/10.1016/j.ijchp.2016.03.002

37. Lee KS, Lennie TA, Wu J-R, Biddle MJ, Moser DK. Depressive symptoms, health-related quality of life, and cardiac event-free survival in patients with heart failure: a mediation analysis. Qual Life Res. $2014 \quad$ Aug 1;23(6):1869-76. Doi: https://doi.org/10.1007/s11136-014-0636-5

38. Bocchi EA, Arias A, Verdejo H, Diez M, Gómez E, Castro $P$, et al. The reality of heart failure in Latin America. J Am Coll Cardiol. 2013 Sep 10;62(11):94958. Doi: https://doi.org/10.1016/j.jacc.2013.06.013

39. Medina LO. Estado del arte de estudios de investigación sobre enfermedad coronaria en la mujer durante el periodo de 1998 hasta el año 2008. Tesis de maestría en enfermería. Universidad Nacional de Colombia. Facultad de enfermería; $2011 . \quad$ Disponible en: http://www.bdigital.unal.edu.co/4875/1/linaorisel medina.2011.pdf

40. Fried LP, Tangen CM, Walston J, et al. Frailty in older adults: evidence for a phenotype. J Gerontol A Biol Sci Med Sci. 2001;56(3):M146-M156. Doi: https://doi.org/10.1093/gerona/56.3.M146

41. Mitnitski A, Rockwood K. Aging as a process of deficit accumulation: its utility and origin. Interdiscip Top Gerontol. 2015;40:85-98. Doi: https://doi.org/10.1159/000364933

42. Afilalo J, Alexander KP, Mack MJ, et al. Frailty assessment in the cardiovascular care of older adults. J Am Coll Cardiol. 2014;63(8):747-762. Doi: https://doi.org/10.1016/j.jacc.2013.09.070

43. Harkness K, Heckman G, McKelvie R. The older patient with heart failure: high risk for frailty and cognitive impairment. Expert Rev Cardiovasc Ther. 2012;10(6):779-95.

Doi:

https://doi.org/10.1586/erc.12.49

44. Lunney JR, Lynn J, Foley DJ, Lipson S, Guralnik JM. Patterns of functional decline at the end of life. Jama. 2003;289(18):2387-92). Doi: https://doi.org/10.1001/jama.289.18.2387

45. Auld JP, Mudd JO, Gelow JM, Hiatt SO, Lee CS. Self-care Moderates the Relationship between Symptoms and Health-Related Quality of Life in Heart Failure. J Cardiovasc Nurs. 2018;33(3):217-24. 
Doi:

https://doi.org/10.1097/JCN.0000000000000447.

46. Salgarriaga $\mathrm{G} \mathrm{Cl}$, Gómez L EA, Navarrete H S, González R G, Gómez M JE. Consenso colombiano para el diagnóstico y tratamiento de la insuficiencia cardiaca. Actualización 2017 Versión de bolsillo. 2017. Disponible en: http://scc.org.co/consensocolombiano-para-el-diagnostico-y-tratamiento-dela-insuficiencia-cardiaca/

47. Lee CS, Mudd JO, Hiatt SO, Gelow JM, Chien C, Riegel B. Trajectories of heart failure self-care management and changes in quality of life. Eur J Cardiovasc Nurs. 2015 Dec;14(6):486-94. Doi: https://doi.org/10.1177/1474515114541730

48. Buck HG, Dickson VV, Fida R, Riegel B, D'Agostino $F$, Alvaro $R$, et al. Predictors of hospitalization and quality of life in heart failure: $A$ model of comorbidity, self-efficacy and self-care. Int J Nurs Stud. 2015 Nov 1;52(11):1714-22. Doi: https://doi.org/10.1016/j.ijnurstu.2015.06.018

49. Riegel B, Carlson B, Moser D, Sebern M, Hicks F, Roland V. Psycometric testing of the self-care of heart failure. J Card Fail. 2004;10:350-60. Doi: https://doi.org/10.1016/j.cardfail.2003.12.001

50. Obiego M, Siennicka A, Jankowska EA, Danel DP. Direction of the relationship between acceptance of illness and health-related quality of life in chronic heart failure patients. J Cardiovasc Nurs. 2017;32(4):348-56. Doi: https://doi.org/10.1097/JCN.0000000000000365

51. Gonzales I, Dos Santos E, Paiva L, Rossi LA, Spadoti RA, Alcalá D. Ansiedad, depresión, resiliencia y autoestima en individuos con enfermedades cardiovasculares. Rev. Latino-Am. Enfermagem. 2016;24:e2836. Doi: https://doi.org/10.1590/1518-8345.1405.2836

52. García JA, García A, López C, Dias PC. Conceptualización teórica de la resilencia psicosocial y su relación con la salud. Salud y drogas [Internet]. 2016;16(1):59-68. Disponible en: https://www.redalyc.org/articulo.oa?id=83943611 006 zer N, Wagner E, Wild B, Frankenstein L, $\mathrm{J}$, Leppert K, et al. Resilience in chronic ıre. Dtsch Med Wochenschr. [Internet] 2014;139(12):580-4.

Doi: https://doi.org/10.1055/s-0034-1369862.

54. Liu M, Wang C, Huang Y, Cherng W, Wang KK. A Correlational Study of Illness Knowledge, Self-care Behaviors, and Quality of Life in Elderly Patients With Heart Failure. J Nurs Res. 2014 Jun 1;22(2):136-45.

Doi: https://doi.org/10.1097/JNR.0000000000000024 